\title{
FARSIDE: A Low Radio Frequency Interferometric Array on the Lunar Farside
}

\author{
Planetary Science and Astrobiology Decadal Survey White Paper
}

Principal Authors: Gregg Hallinan (Caltech), Jack O. Burns (University of Colorado Boulder)

Co-authors: Jim Lux (JPL/Caltech), Andres Romero-Wolf (JPL/Caltech), Lawrence Teitelbaum (JPL/Caltech), Tzu-Ching Chang (JPL/Caltech), Jonathon Kocz (Caltech), Judd Bowman (ASU), Robert MacDowall (NASA Goddard), Justin Kasper (University of Michigan), Richard Bradley (NRAO), Marin Anderson (Caltech), David Rapetti (University of Colorado Boulder), Zhongwen Zhan (Caltech), Wenbo Wu (Caltech), James T. Keane (JPL/Caltech), Mark Panning (JPL/Caltech), Andrew Klesh (JPL/Caltech), Issa Nesnas (JPL/Caltech), Jonathan Pober (Brown University), Steven Furlanetto (UCLA), Alex Austin (JPL/Caltech)

Lead author contact info: Gregg Hallinan gh@astro.caltech.edu, Jack Burns, jack.burns@colorado.edu

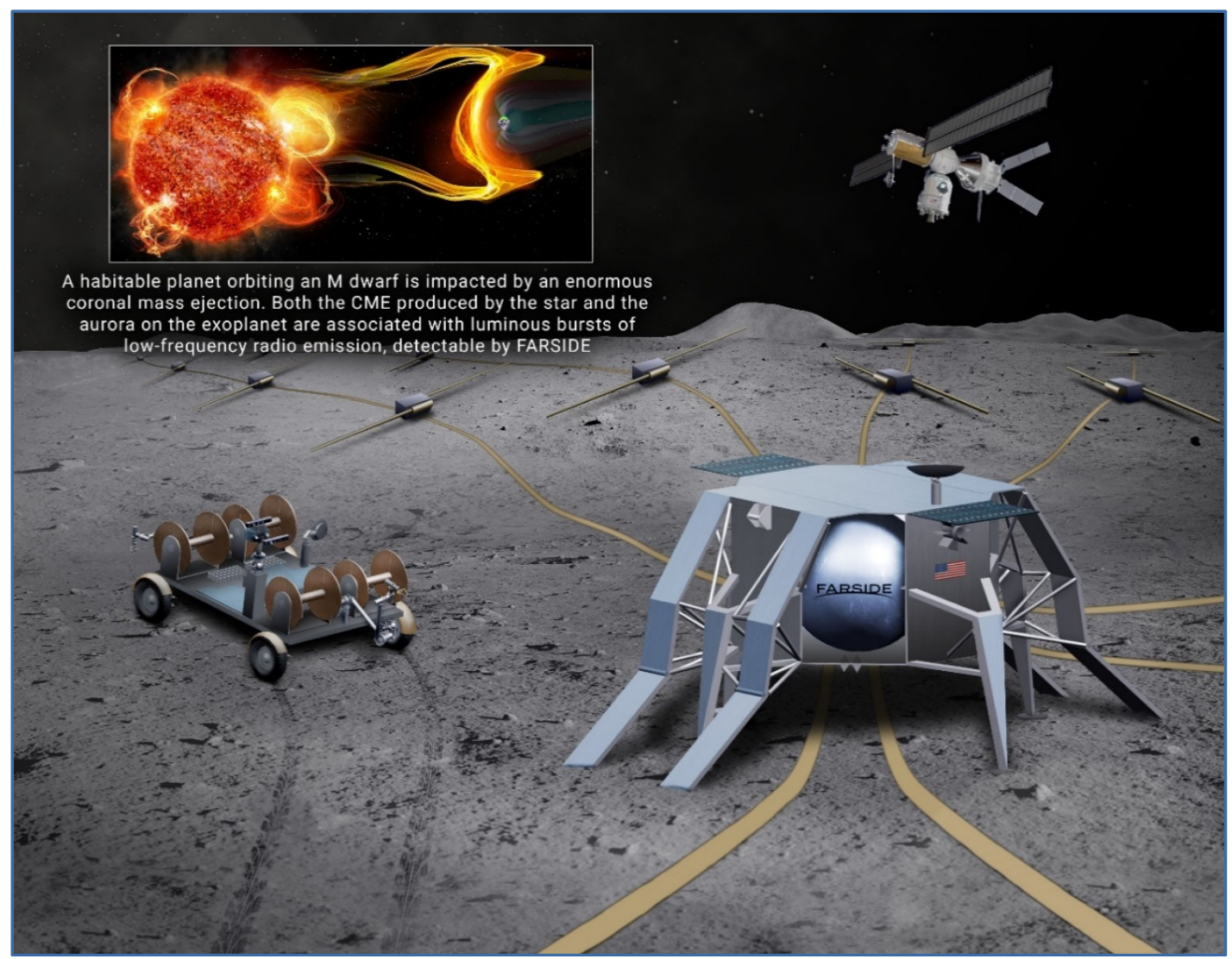

The FARSIDE mission concept consists of a 128-element, radio interferometer, a base station that provides power, signal processing and telecommunications to the Lunar Gateway, and a rover that deploys and assembles the array. The lunar farside provides isolation from interference and is the only location within the inner solar system for sky noise-limited $100 \mathrm{kHz}-40$ $M H_{z}$ radio frequency observations. FARSIDE would also be a unique seismological observatory through the innovative use of Distributed Acoustic Sensing (DAS) technology.

Part of this research was carried out at the Jet Propulsion Laboratory, California Institute of Technology, under a contract with the National Aeronautics and Space Administration.

(C) 2020. All rights reserved. 


\section{EXECUTIVE SUMMARY}

FARSIDE (Farside Array for Radio Science Investigations of the Dark ages and Exoplanets) is a mission concept to place a low radio frequency interferometric array on the farside of the Moon. This mission concept was studied as part of the Decadal Survey on Astronomy and Astrophysics 2020 (Astro2020), in the Astro2020-equivalent to the Planetary Mission Concept Studies program. The NASA-funded design study focused on the instrument, a deployment rover, the lander and base station. The result was an architecture broadly consistent with the requirements for a Probe-class mission in the Astrophysics division or a New Frontiers-class mission in the Planetary Science division [Burns et al. 2019]. This architecture consists of 128 dipole antennas deployed across a $10 \mathrm{~km}$ area by a rover, and tethered to a base station for central processing, power, and data transmission to the Lunar Gateway or a dedicated relay satellite. FARSIDE would provide the capability to image the entire sky each minute in 1400 channels spanning frequencies from $100 \mathrm{kHz}$ to $40 \mathrm{MHz}$, extending down two orders of magnitude below bands accessible to ground-based radio astronomy. The lunar farside is also isolated from terrestrial radio frequency interference, auroral kilometric radiation, and plasma noise from the solar wind. It is thus the only location within the inner solar system from which sky noise limited observations can be carried out at sub-MHz frequencies. FARSIDE would monitor auroral and lightning-generated radio emission from the outer planets and enable the detection of the magnetospheres of the nearest candidate habitable exoplanets. Additionally, the network of hundreds of $\mathrm{km}$ of optical fibers spanning a $10-\mathrm{km}$ diameter area provides an integrated seismological observatory, capable of addressing priority science questions relating to far side moonquakes and the size and properties of the lunar core. Simultaneously, the array will utilize calibration signals from an orbiting satellite to carry out sounding of the mega-regolith and its transition to bedrock at $\sim 2 \mathrm{~km}$ below the lunar surface. For this white paper, we provide a high-level overview of the FARSIDE instrument and mission concept. Further details on the engineering design are found in the NASA mission concept study report (Burns et al. 2019a) and in Romero-Wolf et al. (2020).

\section{KEY SCIENCE GOALS AND OBJECTIVES}

\subsection{Outer Solar System Planets}

All the magnetized planets in our solar system, including Earth, produce bright coherent radio emission at low frequencies, originating in high magnetic latitudes and powered by auroral processes [Zarka 1998]. The emission is due to the electron cyclotron maser instability and is produced at the electron cyclotron frequency $\left(\nu_{\mathrm{ce}} \approx 2.8 \times 10^{6} \mathrm{~B} \mathrm{~Hz}\right.$, where $\mathrm{B}$ is the magnetic field strength in Gauss) enabling precise measurement of the magnetic field strength at the source of the radio emission. It is notable that only Jupiter, with a maximum polar magnetic field strength of $\sim 14$ Gauss, produces radio emission that can penetrate through the Earth's ionosphere. All the other planets (and Ganymede), have magnetic fields of $<2$ Gauss, requiring a space-based observatory for detection. For example, the radio emissions of Saturn, Neptune and Uranus were initially detected in-situ by Voyager during fly-by [Kaiser et al. 1980, Desch et al. 1991, Zarka et al. 1995]. These auroral processes are driven by a) magnetic reconnection between the planetary magnetic field and the magnetic field carried by the solar wind (e.g., Earth, Saturn, Neptune and Uranus), b) the departure from co-rotation with a plasma sheet residing in the planetary magnetosphere (e.g., Jovian main auroral oval) or c) interaction between the planetary magnetic field and orbiting moons (e.g., Jupiter-Io current system) [Zarka 1998 and references therein, Bagenal \& Dols 2020]. As well as providing diagnostic information on the presence, strength and extent of planetary magnetospheres, the detected radio bursts are the only means to accurately determine the rotation period of the planetary interior for the gas giants. FARSIDE will be the first radio telescope with sufficient sensitivity to detect the radio emission from all the magnetized 
planets from the distance of the EarthMoon system. This will enable monitoring of the impact of the variable solar wind on auroral radio flux density, including during interplanetary shocks from coronal mass ejections (CMEs). Long-term monitoring of the Kronian radio emission period, modulated by the solar wind, will refine measurement of the true planetary rotation period [Zarka et al. 2007]. Neptune and Uranus have not been observed at radio wavelengths since Voyager, with a number of questions remaining unresolved [white paper by Kollmann et al.].

Saturn and Uranus have also been detected by Voyager to produce radio emission associated with atmospheric lightning [Zarka et al. 2004] (Figure 1). This radio emission can potentially inform on atmospheric dynamics and composition, when compared to optical imaging of cloud data. These emissions are also easily detectable by FARSIDE (Figure 1).

Finally, FARSIDE would offer the unique possibility of searching for radio emission from large bodies beyond Neptune out to 100s of AU. This includes, but is not limited to, the putative Planet 9 [Batygin \& Brown 2016].

\subsection{The Magnetospheres of Habitable Planets}

The discovery of life on a planet beyond Earth and even outside our solar system is at the heart of NASA's Science Mission Directorate. It is becoming increasingly apparent that both the selection of candidate exoplanets for deep searches for biosignatures, and interpretation of the observed atmospheric composition, must take into account the space weather environment of the host star, and whether the planet possesses a large-scale magnetosphere capable of retaining an atmosphere within this space environment [Lammer et al. 2007]. The detection of radio emission is the only known method to directly detect the presence and strength of the magnetospheres of candidate habitable planets. Detection of the magnetic field of planets orbiting in the habitable zone of $\mathrm{M}$ dwarfs (cool stars with masses $<50 \%$ of the Sun) is particularly key, as such planets are most abundant in the solar neighborhood and may require a significantly stronger magnetosphere than Earth to sustain an atmosphere [Lammer et al. 2007].

However, extending to the exoplanet domain requires a very large collecting area and the detection of the magnetic fields of candidate habitable planets will almost certainly require a space-based array if 
the magnetic fields are within $10 \times$ of Earth's magnetic field. It is possible to estimate the expected radio power from such planets, based upon scaling laws known to apply to radio emission from the solar system planets. These scaling relations are not only descriptive but predictive, with the luminosity of both Uranus and Neptune predicted before the Voyager 2 encounters and found to be in good agreement with the measurements [Desch and Kaiser 1984; Desch 1988]. Extrapolations to terrestrial exoplanets in the habitable zone of $\mathrm{M}$ dwarfs, and thus embedded within a dense stellar wind environment, predict radio luminosities that are orders of magnitude higher than the Earth [Vidotto et al. 2019; Grießmeier 2015]. During enhanced solar wind conditions, the Earth's radio emission can increase in luminosity by orders of magnitude (Figure 2) and a similar effect is expected for exoplanets. It is essential to have the capability to monitor large numbers of planets simultaneously to detect periods where exoplanets greatly increase in luminosity.
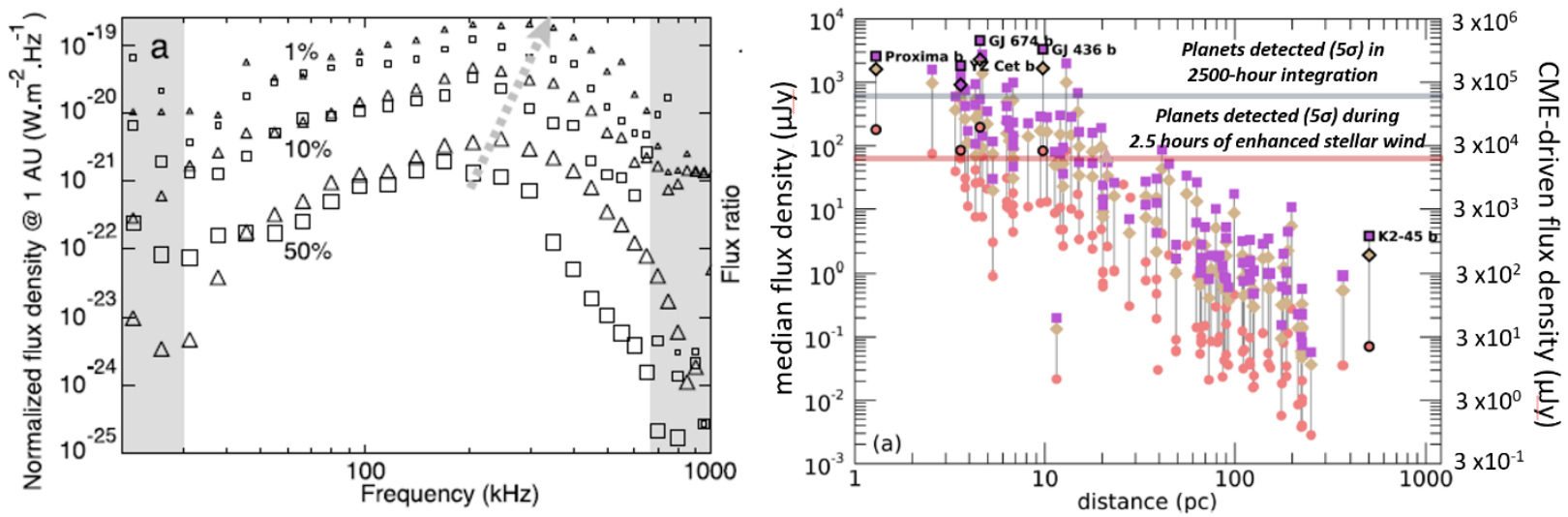

Figure 2. Left: The radio spectrum of Earth's auroral kilometric radiation for 50\%, 10\% and 1\% of the time. The flux density across the entire detectable band can vary by factors of a few hundred [Lamy et al. 2010]. Right: The predicted average flux radio density at $\sim 280 \mathrm{kHz}$ of known exoplanets orbiting M dwarfs from Vidotto et al. [2019], assuming a magnetic field of 0.1 G (10\% of Earth's magnetic field). Purple, yellow and red data points reflect different models for the quiescent stellar wind. The horizontal grey line represents the $5 \sigma$ detection limit (y-axis on left-hand side) on the median flux density for the auroral radio emission detectable from FARSIDE in a 2500-hour integration. The horizontal red line represents the $5 \sigma$ detection limit (y-axis on right-hand side) in a 2.5-hour integration during enhanced stellar wind conditions, e.g., during a CME, assuming a similar degree of variability as observed for Earth's auroral kilometric radiation. A large sample of planets, including some nearby candidate habitable planets (such as Proxima b), are detectable.

FARSIDE will image 10,000 $\mathrm{deg}^{2}$ of sky visible from the lunar far side every 60 seconds, in 1400 channels spanning $150 \mathrm{kHz}-40 \mathrm{MHz}$. The sensitivity at the lower end of the band, enabled by the unique environment of the lunar far side, is particularly well suited to the search for radio exoplanets. In each FARSIDE image, there will be $\sim 2,000$ stellar/planetary systems within 25 pc monitored simultaneously. An advantage at these low frequencies, is that the galaxy is optically thick, and thus long integrations are possible without classical confusion noise. Over the course of a 2-year observing program, FARSIDE will have accumulated an average of 4,000 hours of observing time on each of 8,000 stellar planetary systems within 25 pc. Radio exoplanets will be identified by two means:

i) 2,500 hour integrations will measure the average radio power from exoplanets.

ii) 2.5-hour integrations will measure heightened emission during CME interaction.

Figure 2 highlights that FARSIDE would detect the radio emission from a population of Earth-sized, and super-Earth sized planets orbiting nearby $\mathrm{M}$ dwarfs, including a number of candidate habitable planets, providing the first measurements of terrestrial planet magnetospheres outside our solar system. The radio emission can be distinguished from that of the star by rotational modulation, as well 
as circular polarization which is largely absent for interplanetary radio bursts. Detection of magnetospheres, if present, would identify the most promising targets for follow-up searches for biosignatures, as well as providing a framework for comparative analysis of exoplanet magnetospheres and atmospheric composition.

\subsection{Lunar Quakes and Core with FARSIDE Optical Fibers}

Tectonic activity and internal structure of planets/moons are fundamental to understanding their formation, evolution and habitability. The moon is of particular interest, due to its close relation with the Earth [Canup 2012]. Four seismometers deployed during the Apollo missions provided fundamental constraints on the Moon activity and structure [Nakamura et al. 1982, Weber et al. 2011, white paper by Weber et al.]. However, outstanding scientific questions, such as the mantle structure and seismic activity at the far side and the existence of a liquid core, remain unresolved. Answering these questions requires more seismometers, desirably dense seismic arrays, on the far side. Constrained by technical difficulties and cost, planetary seismology has mostly been focusing on single devices (e.g., Mars InSight) or a few stations (e.g., Apollo, Lunar Geophysical Network).
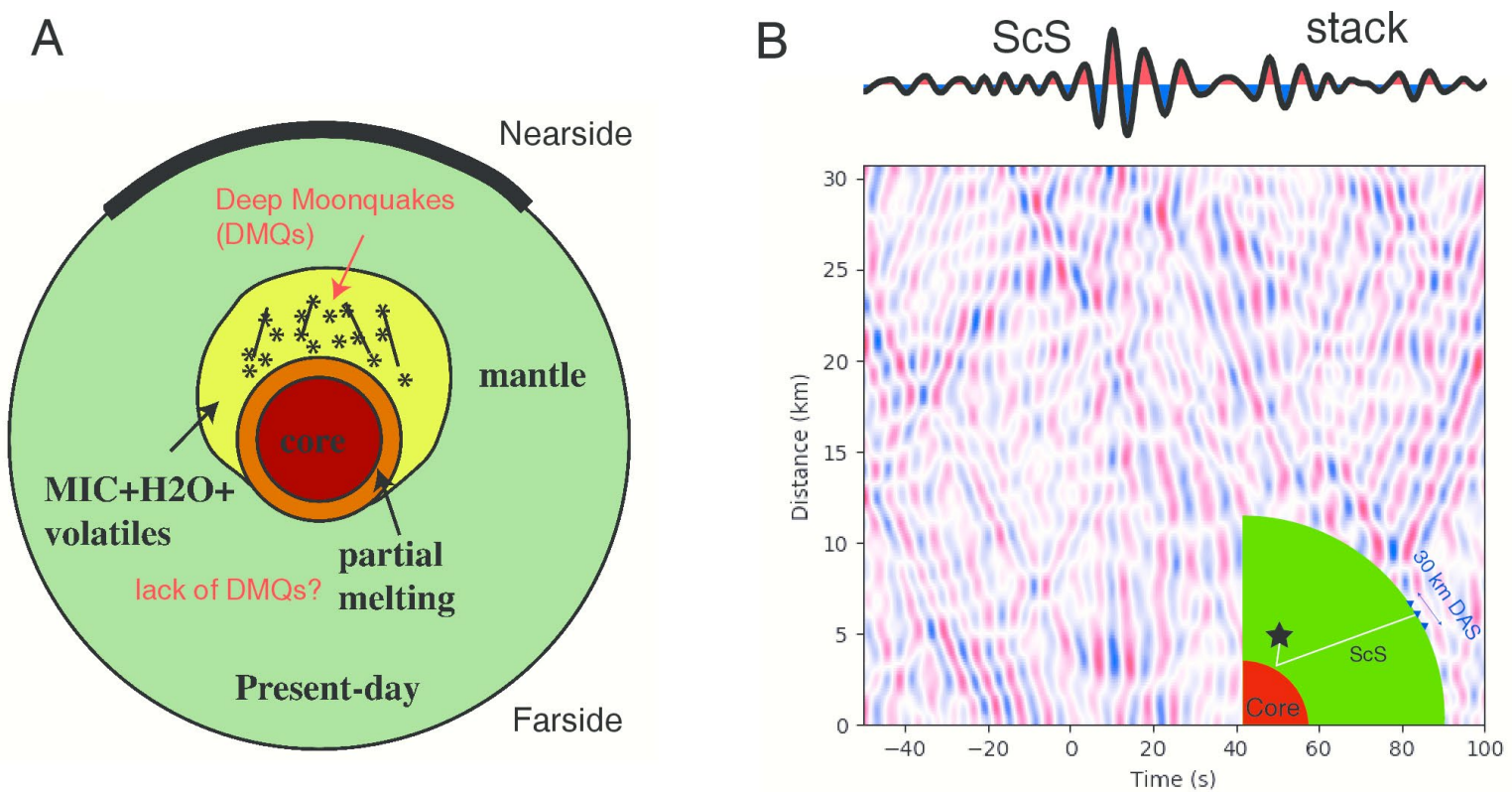

Figure 3. Hypothetic hemispherical lunar structure and a synthetic example of DAS array technique. (A): A schematic representation of the hemispherical lunar structure. Adapted from Qin et al. [2012]. (B): A synthetic example of retrieving core phase $\mathrm{ScS}$ from a 30-km long DAS array. The strongly scattered seismic waves recorded over the DAS array completely mask the weaker ScS core phase (ray path shown in the inset), but array stacking enhances the signal as shown in the top panel. Time zero is the ScS arrival time predicted by the 1D model [Garcia et al. 2011].

The $\sim 50-\mathrm{km}$ long fiber optic cables laid down by FARSIDE could enable the deployment of a 10-km aperture, 5000-sensor seismological observatory on the far side of the Moon, with minimum additional cost. The Distributed Acoustic Sensing (DAS) technology can turn every few meters of a 10 's of $\mathrm{km}$ long optical fiber into a seismic sensor, by attaching an interrogation unit to one end of the fiber [Zhan 2019]. DAS works by shining a laser pulse into the fiber from one end and interrogating the "echo" of Rayleigh scattering from intrinsic fiber defects. If a fiber section is strained, the relative positions of the fiber defects within the section will change, and so will the corresponding backscattering, in both amplitude and phase. DAS measures the changes at a high sampling rate (e.g., $10 \mathrm{kHz}$ ) and converts them to strain measurements. The technology has proven to work well on Earth 
with either dedicated fiber optic cables or existing telecom fibers, without interfering data transfer over other strands of fiber in the same cable.

The seismological component of FARSIDE will require a few more strands of fiber in the cables connected in a continuous loop, and additional payload of DAS instrument at the FARSIDE Base Station. It can potentially share systems of power supply, environmental protection, and data processing/telemetry with the radio telescope component. Current commercial DAS instruments weigh about $50 \mathrm{~kg}$ and consume $200 \mathrm{~W}$ power, most of which is related to the computing units. A space qualified FPGA solution to the data processing could substantially reduce the weight and power consumption. It is thus a possible low-impact future augmentation of the FARSIDE baseline design.

The FARSIDE seismic network will answer two key science questions among many others:

Deep moonquakes at the far side: The Apollo seismometers detected thousands of moon quakes at 700-1100 km depth from the lunar nearside, but much fewer from the far side [Nakamura 2005]. This lack of far-side events could be due to detection bias with all the sensors on the near side, or a true hemispheric difference in lunar deep structure and dynamics, which would have critical implications to lunar evolution [Qin et al. 2012; Laneuville et al. 2013] (Figure 3). It is well known that shallow lunar structure has clear hemispheric asymmetry, but the depth extent of which is still unclear. To maximize the detection capability, we can apply array-processing techniques to the FARSIDE seismic network and enhance data signal-to-noise ratios by tens of times.

Size and properties of the lunar core: Reflections from the lunar core-mantle boundary have been difficult to observe, due to scattering in the highly heterogeneous lunar crust [Weber 2011]. Therefore, there are still large uncertainties on the lunar core size and properties. Does the Moon have liquid outer core and a solid inner core like the Earth? Constraining the structure of core is almost always the top goal of planetary geophysical program due to its importance to understanding planetary evolution and habitability. Previous studies on Earth and synthetic simulations for the Moon have demonstrated that the $10-\mathrm{km}$ aperture FARSIDE array would greatly enhance the lunar core phases hidden in scattered waves and provide a clear picture of the lunar core (Figure 3).

Potential for active source surveying: With the addition of a vibrational source on or near the base station or deployed in a targeted location along the deployed cables, investigations of upper crustal structure of the moon could also be performed. Explosive sources recorded at an array of geophones were used to constrain the upper $10 \mathrm{~km}$ of the lunar crust at the Apollo 17 landing site [Kovach and Watkins 1973], and a range of techniques are available for performing active source surveying in planetary settings today [Courville et al. 2020]. The most promising geologic targets due to their relevance to human exploration for active source surveying on the Moon would be polar ice deposits [e.g. Hayne et al. 2020] or lava tubes associated with orbital observations of sinuous rilles and skylights and nearly linear gravity anomalies [e.g. Chappaz et al. 2017] which are associated with mare deposits; however, neither of these is likely at ideal FARSIDE locations. Detailed stratigraphy of the subsurface, however, remains a valuable product of active source approaches and provides a complementary approach to radar sounding performed with the array.

\subsection{Sounding of the Lunar Sub-surface}

FARSIDE has the potential to sound the mega-regolith and its transition to bedrock expected at $\sim 2$ $\mathrm{km}$ below the surface [Hartmann 1973]. The Lunar Radar Sounder (LRS) onboard the KAGUYA (SELENE) spacecraft has provided sounding observations of the lunar highlands [Ono et al. 2010] and found potential scatterers hundreds of meters below the subsurface. However, the results are inconclusive due to surface roughness. FARSIDE, by virtue of being on the surface, would not be affected by roughness. Data from a calibration beacon in orbit would be synthesized to identify deep scatterers and the transition to bedrock at $\mathrm{km}$ depths by virtue of the low frequencies, which are 
significantly more penetrating. Deep subsurface sounding can also be performed passively using Jovian bursts from $150 \mathrm{kHz}$ to $20 \mathrm{MHz}$ [e.g., Romero-Wolf et al. 2015]. The array would cover a $10 \mathrm{~km} \times 10 \mathrm{~km}$ area on the lunar highlands providing a 3-D image of the highland subsurface structure.

\section{TECHNICAL OVERVIEW}

As a low frequency radio observatory $(100 \mathrm{kHz}-40$ $\mathrm{MHz}$ ) targeting faint signals, the FARSIDE array is required to be above the Earth's plasmasphere and to be shielded from radio frequency interference (RFI). Ground-based radio astronomy is limited to $>10 \mathrm{MHz}$, due to attenuation in the ionosphere. Moving above the atmosphere opens an additional 2 orders of magnitude in frequency range below which observations become limited by the plasma density of the solar wind at Earth orbit. However, the sensitivity of an Earth-orbiting radio telescope is increasingly limited below $\sim 1 \mathrm{MHz}$. This is partially due to the presence of anthropogenic

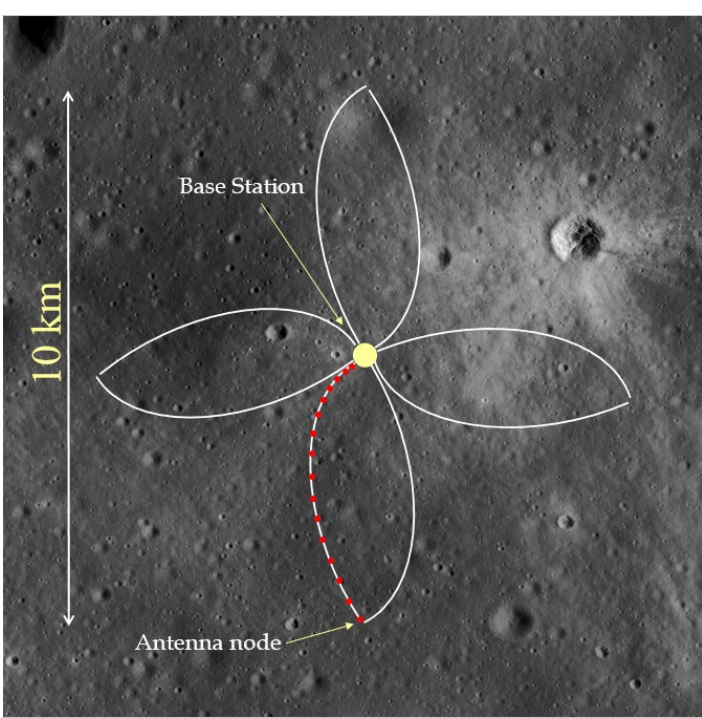

Figure 4: FARSIDE will consist of 128 antenna nodes deployed by a rover from a central base station, arranged in a petal configuration. radio frequency interference $(\mathrm{RFI})$ and the intense auroral kilometric radiation (AKR) produced by Earth. More fundamentally, the system noise below $1 \mathrm{MHz}$ in Earth orbit is limited by the electrons in the solar wind colliding with the antenna and inducing currents [Meyer-Vernet \& Perche 1989]. Together, these factors prevent sensitive searches for planet and exoplanet radio emission below $1 \mathrm{MHz}$. By contrast, the lunar farside provides a unique environment for radio astronomy within the inner solar system, with $>100 \mathrm{~dB}$ attenuation of both RFI and AKR [Bassett et al. 2020]. Most importantly, a plasma cavity exists on the surface, particularly on the night-time side, that provides sufficient isolation for sky noise dominated observations down to $\sim 200 \mathrm{kHz}$.

The FARSIDE instrument front-end uses electrically short simple dipole antennas to achieve sky background noise-limited observations. The regolith in the lunar highlands is thick, has low conductivity, and varies slowly with depth removing the need for a ground plane. Calibration will make use of astronomical sources, as well as an orbiting calibration beacon that will map each antenna gain pattern together with the effect of the lunar subsurface. The implementation uses thin wires on the ground with the rest of the system leveraging existing designs: high space heritage front-end amplifiers, fiber optics, and digital hardware.

The FARSIDE mission includes three components: a commercial lander (e.g., part of NASA's Commercial Lunar Payload Services) carrying the FARSIDE base station and a rover which deploys the array of antenna nodes. The base station includes a pair of enhanced Multi Mission Radioisotope Thermoelectric Generators (eMMRTG) to provide power for the receivers and the signal processing functions of the FX correlator, as well as communicating with the Lunar Gateway (or a similar data relay capability from the lunar farside) to send the science data back to Earth. The rover will be solar powered, hibernating at night, and includes a telecommunications subsystem to communicate with the Gateway during the array deployment operations phase. The 128-node array is configured in 4 petals to minimize rover travel on the lunar surface, with a deliberate asymmetry to improve imaging performance. The array is deployed on 4 successive lunar days, one petal of 32 nodes deployed in the 10-14 days of sunlight, with the rover hibernating during lunar night. The FARSIDE mission architecture and concept of operations were developed in a sequence of studies conducted at the Jet 
Propulsion Laboratory (JPL) by JPL's concurrent design facility, Team X. The sequence of Team X studies assessed the technical feasibility of the instrument, the mission as a whole, and the cost realism for implementation as a Probeclass mission. The pre-reserve total cost for the mission (Phase $\mathrm{A}-\mathrm{F}$ ) is $\sim \$ 1.1 \mathrm{~B}$, including the launch vehicle and the nuclear material power sources for the base station and the 128 receiver elements comprising the science instrument. The development cost (Phase A-D) is $\$ 800 \mathrm{M}, \sim \$ 110 \mathrm{M}$ for the instrument (payload) and $\sim \$ 510 \mathrm{M}$ for the flight system, which consists of the base station and deployment rover integrated into a large lunar lander. The Blue Origins Blue Moon Lander was selected as a reference lander for the

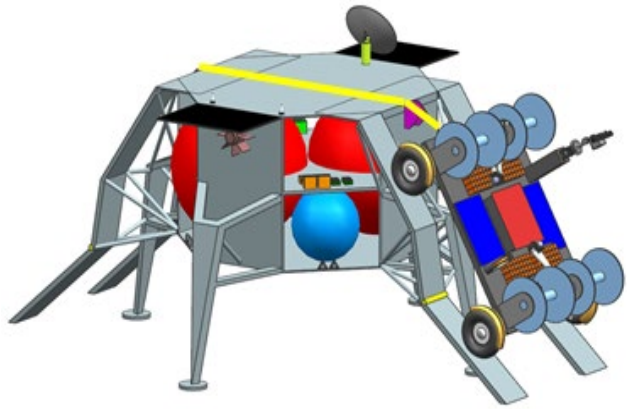

Figure 5: Conceptual design of the FARSIDE rover being deployed from the Base Station. design.

FARSIDE is timely. Increasing interest in lunar exploration from NASA is driving the development of the Lunar Gateway and supporting technology and stimulating the advancement of progressively more capable lunar landers. FARSIDE will take advantage of these investments from NASA's Artemis program, which are expected to reach sufficient maturity by the mid-2020's to support a mission in the 2025-2035 time frame. The FARSIDE instrument and the computing elements on the base station will need modest engineering development; no new technology is required. Finally, FARSIDE would address priority science questions across the NASA Science Mission Directorate, including the Astrophysics Division (exoplanets, Dark Ages cosmology) and Planetary Science Division (lunar interior, gas giant magnetospheres) and Heliophysics Division (solar flares, CMEs, solar radio bursts).

\section{REFERENCES}

- Bagenal, F., \& Dols, V. 2020, JGR, 125(5), e27485 - Bassett N., et al. 2020, AdSpR, 66, 1265 Batygin K., Brown M.E., 2016, AJ, 151, 22 - Burns J.O. et al., 2019, arXiv, arXiv:1911.08649 — Canup R.M., 2012, Sci, 338, 1052 - Chappaz L., 2017 et al., GeoRL, 44, 105 - Courville S.W., Putzig N.E., Sava P.C., Mikesell T.D., Perry M.R., 2020, LPICo, 2241, 5055 - Desch M.D., 1988, GeoRL, 15, 114 - Desch M.D., Kaiser M.L., 1984, Natur, 310, 755 a Grießmeier J.-M., 2015, ASSL, 213 п Desch M.D. et al. 1991, uran.book, 894 - Hayne P.O. et al., 2020, LPICo, 2241, 5136 - Hartmann, W.K. 1973, Icarus, 18, 634 - Kovach R.L., Watkins J.S., 1973, Sci, 180, 1063 - Lammer H. et al., 2007, AsBio, 7, 185 - Kaiser M.L et al. 1980, Sci, 209, 1238 - Laneuville M. et al. 2013, JGRE, 118, 1435 - Lamy L. et al. 2010, JGRA, 115, A09221 - Meyer-Vernet N. \& Perche C., 1989, JGR, 94, 2405 Nakamura Y., Latham G.V., Dorman H.J., 1982, LPSC, 87, 117 - Ono T. et al., 2010, SSRv, 154, 145 - Qin C., Muirhead A.C., Zhong S., 2012, Icar, 220, 100 - Nakamura Y., 2005, JGRE, 110, E01001 - Romero-Wolf, A. et al. 2015, Icarus, 248, 463 - Vidotto A. A., Feeney N., Groh J.H., 2019, MNRAS, 488, 633 - Weber R.C. et al. 2011, Sci, 331, 309 - Zarka P. et al. 1995, netr.conf, 341 - Zarka, P. 1998,JGR,103, 20159 - Zarka P. et al. P\&SS, 52, 1435 - Zarka P., 2007, P\&SS, 55, 598 — Zhan Z., 2019, GeoRL, 46, 4747 